\title{
Dark Tetrad Personality Traits and the Propensity to Lie Across Multiple Contexts
}

\author{
Loch Forsyth $^{1}$, Jeromy Anglim ${ }^{1}$, Evita March ${ }^{2}$, Barbara Bilobrk ${ }^{2}$ a
}

\begin{abstract}
The current study investigated the relationships between the Dark Tetrad traits (i.e., Machiavellianism, narcissism, psychopathy, and sadism) and the propensity to lie in general and across the domain-specific contexts of academic, relationship, and professional scenarios. Participants $(N=615 ; 61 \%$ female: age $M=26.83, S D=8.36)$ completed the Short Dark Triad and Short Sadistic Impulse Scale. They then read and responded to vignettes in three separate contexts (relationship, academic, and professional) and indicated their propensity to lie, lying efficacy, perceived cognitive load, and emotional response to lying. As hypothesized, individuals higher on the Dark Tetrad were found to report a higher propensity to lie. The Dark Tetrad traits were correlated with less cognitive load, more positive affect, and less negative affect while lying. Of the four traits that comprise the Dark Tetrad, Machiavellianism was the strongest predictor across each of the domain specific contexts. Sadism and narcissism both presented as significant and somewhat comparable predictors to deceive. The findings from the current study highlight the dangers of defaulting to truth-based judgements when engaging in interactions involving those high on the Dark Tetrad.
\end{abstract}

Keywords: dark triad, dark tetrad, deception, lying, personality

\section{Introduction}

People have the opportunity to lie to others in a range of different contexts. Lies are a form of deception involving an "outright falsehood, which is consciously known to be false by the teller and is not signaled as false to the message recipient." (Levine, 2020, p. 94). While individuals report lying often in their daily lives (DePaulo, Kashy, Kirkendo, Wyer, \& Epstein, 1996), evidence suggests that the majority of lies are told by a few prolific liars (George \& Robb, 2008; Serota Levine, \& Boster, 2010). Habitual deception may result in a range of consequences and the erosion of trust in any future interactions (DePaulo, 2019; Griffin \& Bender, 2019). Specifically, the destructive nature of deception can impact the integrity of organizational and academic processes requiring the need for methods that can identify those more likely to engage in deceptive behavior (Porter \& ten Brinke, 2010). Personality screening is one potential approach that could assist in identifying those who have a higher natural propensity to deceive across interpersonal contexts (Bonaccio, Reeve \& Lyerly, 2016; Jones \& Paulhus,

\footnotetext{
a Please cite as:

Forsyth, L., Anglim, J., March, E., \& Bilobrk, B. (in press). Dark Tetrad Personality Traits and the Propensity to Lie Across Multiple Contexts. Personality and Individual Differences, 177, 110792. https://doi.org/10.1016/j.paid.2021.110792

${ }^{1}$ School of Psychology, Deakin University, Geelong, Australia ${ }^{2}$ Federation University, Ballarat, Australia; Correspondence concerning this article should be addressed to Loch Forsyth, School of Psychology, Deakin University, Locked Bag 20000, Geelong, 3220 Australia. Email: 1.forsyth@deakin.edu.au . Funding statement: This research did not receive any specific grant from funding agencies in the public, commercial, or not-for-profit sectors. Data, scripts, and materials are available on https://osf.io/cw2b7
} 
2011). Nonetheless, limited research has examined whether personality traits can predict the propensity to lie across a range of trust-based contexts where truthful interactions are imperative for the overall integrity of the context in question. In the current paper, we explore the utility of the Dark Tetrad personality traits to predict deception across a range of contexts, including relationships, professional contexts, and academia.

\subsection{The Dark Tetrad and Deception}

Originally a Dark Triad of personality (subclinical narcissism, Machiavellianism, and psychopathy; Paulhus \& Williams, 2002), the Dark Tetrad also includes trait sadism (Chabrol et al., 2020; Chabrol et al., 2015). Although these dark personality traits share some overlap (e.g., disagreeable, socially aversive), they are considered conceptually distinct (Chabrol et al., 2009). Trait narcissism is characterized by egoism, dominance, and self-centeredness (Carpenter, 2012; Jonason \& Krause, 2013), trait Machiavellianism is characterized by cynicism, strategic manipulation, and exploitation of others (Abell \& Brewer, 2014; Jonason, Li, Webster, \& Schmitt, 2009), trait psychopathy is characterized by a callous nature, empathy deficits, and impulsivity (Miller \& Lynam, 2015; Venables, Hall, \& Patrick, 2014), and trait sadism is characterized by enjoying causing others psychological or physical harm (Chabrol et al., 2009). Despite these dark traits being highly antisocial, researchers have argued that they also have an adaptive purpose (see Jonason \& Webster, 2012; Jonason, Webster, Schmitt, Li, \& Crysel, 2012); in particular, they are associated with self-interest and the exploitation of others for one's own needs (O'Boyle, Forsyth, Banks, \& McDaniel, 2012).

Deception is understood to be a central component of dark personality traits, particularly the Dark Triad (Jonason, Lyons, Baughman, \& Vernon, 2014). Individuals who score higher on the Dark Triad traits have been found to tell more lies (Jonason et al., 2014), report believing they have a higher ability to lie, and also report a higher preparedness to deceive others (Wright, Berry, Catmur, \& Brid, 2015). Importantly, because humans are generally poor at identifying deception (Bond \& DePaulo, 2006), many lies go undetected. The dark traits have been linked to a variety of deceptive behaviors including academic dishonesty (Brunell, Staats, Barden, \& Hupp, 2010), relationship infidelity (Brewer, Hunt, James, \& Adell, 2015), and manipulative behavior (Raskin, Novacek, \& Hogan, 1991; Rauthmann, 2012). Baughman, Jonason, Lyons, \& Vernon (2014) found all three Dark Triad traits predicted a propensity to lie in an academic context, while only psychopathy and Machiavellianism predicted the probability to lie in a mating context.

Compared to the Dark Triad, the relationship between the Dark Tetrad and deception remains comparatively underexplored. Despite this paucity in research, there are reasons to expect sadism to predict greater deception, as research has shown sadism to be a significant, strong predictor of Internet trolling (Craker \& March, 2016) which is defined as a deceptive online behaviour (Hardaker, 2010).

\subsection{Deception Across Contexts: Professional, Academic, and Relationships}

To understand the relationship between dark personality traits and deception across a range of social contexts, we can apply social exchange theory (see Cropanzano \& Mitchell, 2005, for an overview). Social exchange theory has previously been adopted to explore the relationship between the dark personality traits and violations of 
these social norms of trust and reciprocity in professional contexts (see O'Boyle et al., 2012). Social exchange theory posits that social relationships are initiated and sustained through the reliable exchange of rewards and the imposition of costs between individuals, and these exchanges build trust and reciprocity in relationships. In social exchanges, deception is employed as a strategy to obtain and/or maintain personal resources and rewards (Marelich et al., 2008). It follows then that individuals who are more likely to seek personal gain in social exchanges (see Jonason et al., 2010) may be more inclined to adopt deceptive practices. In accordance with social exchange theory, we propose that individuals with high levels of dark personality traits will be more likely to adopt deceptive practices across a range of contexts. the Further, we attribute this relationship to characteristics that are typical of each dark trait . For example, the distrust inherent in Machiavellianism, egoism in narcissism, callousness in psychopathy, and the sadistic enjoyment of harming others may lead these individuals to violate trust and reciprocity norms established via social exchanges.

In addition to professional contexts, researchers have explored the relationship between dark personality traits and deception in more intimate relationships. In particular, this relationship is attributed to an exploitative mating style that is characteristic of the dark personality traits (Jonason, Li, \& Buss, 2010). Specifically, individuals with high levels of these dark personality traits exhibit greater preference for more short-term, casual sex relationships, and this preference may foster more exploitative mating styles, including deception (Jonason, Li, Webster, \& Schmitt, 2009). Such deception in mating strategies (and by extension, relationships) could therefore be an adaptive tactic and facilitates mating success (Baughman, Jonason, Lyons, \& Vernon, 2014).

\subsection{The Current Study}

Although research (albeit limited) has explored the relationship between dark personality traits and lying propensity in different contexts, these studies typically focus on contexts independently. To date, only one study (Baughman et al., 2014) has investigated the relationship between dark personality traits and the propensity to lie across various contexts, specifically academic and mating (i.e., relationship) contexts. In the current study, we expand on previous studies by investigating the effects of the Dark Tetrad on the propensity to lie generally and across the multiple domain specific contexts of academic, relationships, and professional contexts. As such it also provides the first study to examine the ability of trait sadism to predict deception across a range of contexts (i.e., relationships, professional, and academic). The study also examines how dark traits relate to aspects of the lying process including efficacy, cognitive load, and affect. It was hypothesized that the Dark Tetrad traits would predict a higher propensity to lie across academic, relationship, and professional contexts.

\section{Method}

Data, scripts, and materials are available on https://osf.io/cw2b7

\subsection{Participants and Procedure}

An online convenience sample of English-speaking adults were recruited through online forums including Reddit, Facebook, and LinkedIn in July 2019. Participants completed an online survey that included demographics, measures of the 
Dark Tetrad, items measuring general lying tendencies, and the three vignettes. The study received ethics approval from the fourth author's institutional ethics review board.

The cleaned sample consisted of 615 participants $(60.5 \%$ female; $37.1 \%$ male; $2.4 \%$ other) with a mean age of 26.83 years $(S D=8.36)$. Country of residence was broadly similar to the distribution of English-speaking Internet users (i.e., mostly US, UK, Australia, Canada, etc.; see online supplement for further demographic details). This was based on an initial sample of 632 of which participants were removed for one or more of the following reasons: disagreeing or strongly disagreeing with more than 31 of the Tetrad items $(n=1)$, agreeing or strongly agreeing with more than 31 of the Tetrad items $(n=0)$, providing the neither agree not disagree response to more than 15 of the Tetrad items $(n=6)$, or completing the study too quickly (defined as 286 seconds, $n=12$ ). The study had $80 \%, 95 \%$, and $99 \%$ power to detect population correlations of $.113, .145$, and .171 respectively.

\subsection{Materials}

\subsubsection{Dark Tetrad Personality}

The Dark Triad traits of narcissism, Machiavellianism, and psychopathy were measured using the 27-item Short Dark Triad (SD3; Jones \& Paulhus, 2014). Sadism was measured using the 10-item Short Sadistic Impulse Scale (SSIS; O'Meara, Davies, \& Hammond, 2011). Items for both measures employed a 5-point scale from $1=$ Strongly Disagree to 5 = Strongly Agree. Scale scores were the mean after any item reversal. Cronbach's alphas for the sample were .81 (Machiavellianism), .75 (narcissism), .64 (psychopathy), and .88 (sadism).

\subsubsection{General Lying Tendencies}

General lying tendencies were measured using three statements regarding their typical lying behaviors. General propensity to lie was based on the response to the question "How often do you lie?" General lying efficacy was measured as the average of "How often are your lies detected?" (reversed) and "Please indicate the degree to which you feel people find your lies believable?". All items were on a 7-point scale where $1=$ Never, $4=$ Rarely and $7=$ Always. The alpha for general lying efficacy was .69 .

\subsubsection{Scenarios}

Participant were presented with three vignettes that provided three contexts for lying: Relationships, academic, and professional (see Online Supplement for text). The order of the vignettes was randomized. The relationship and academic contexts were taken from Baughman et al. (2014), while the professional context was developed for this study. After reading each context the participants were asked several questions also taken from Baughman et al. (2014). Propensity to Lie was measured using the single item that assessed how likely they would lie, and Lying Efficacy was assessed as the perceived likelihood that the person lied to would believe the lie. Both questions were on a 7-point scale from $1=$ Not at all, $4=$ Somewhat and $7=$ Completely. The Cognitive Load scale consisted of 6 items on a 5-point scale from $1=$ strongly disagree to $5=$ strongly agree. Items assessed the required preparation, the difficulty, and the effort required to lie. Scale scores were the mean of items. Positive Affect and Negative Affect were measured by assessing the degree to which participants would expect to experience a range of emotions in the context. This included 5 positive emotions (i.e., joy, 
happiness, amusement, satisfaction, enjoyment) and 5 negative emotions (i.e., anger, guilty, annoyance, disgust, fear). Scale scores were the mean after reversing negative emotions. These fives scales were computed separately for each scenario. In addition, scenario averages were obtained for the five scales by taking the mean scale score. Cronbach's alphas of these scenario averages were .54 (propensity to lie), .60 (Lying efficacy), .72 (Cognitive Load), .76 (Positive Affect), .79 (Negative Affect).

\section{Results}

\subsection{Overall Correlations}

Table 1 presents correlations and descriptive statistics between demographics, traits, general lying tendencies, and responses to the scenarios. Several key observations can be made. First, the Dark Tetrad traits were all positively correlated. In general, Machiavellianism, Psychopathy, and Sadism were more intercorrelated than was Narcissism. Second, women were slightly lower on all Dark Tetrad traits, whereas age was only correlated with Machiavellianism $(r=-.12)$. Third, general lying efficacy was only weakly correlated with general propensity to lie $(r=.20)$. The Dark Tetrad correlated well with both propensity to lie and lying efficacy, but correlations were larger with propensity to lie than lying efficacy. The one instance where this differential correlation was least pronounced was for narcissism. Of the Dark Tetrad, the strongest correlate of propensity to lie was Machiavellianism $(r=.43)$.

Several interesting findings also emerged with average responses to the scenarios. In general, means scores for propensity to lie and lying efficacy were relatively low considering the 7-point scale. People generally reported experiencing very little positive affect, but some negative affect. There were no correlations between age or gender with propensity to lie. The main demographic effects were that women experienced less pleasant affect when lying (less positive, more negative) and older people reported less cognitive load. Correlations between the Dark Tetrad and propensity to lie were very similar to those with the stated general tendency with Machiavellianism once again being the largest correlate. The Dark Tetrad traits were correlated with reduced cognitive load while lying. With regards to the emotional experience of lying Dark Tetrad traits were associated with a strong tendency to experience greater positive affect and a more modest tendency to experience less negative affect.

\subsection{Scenario Analysis}

Table 2 presents the descriptive statistics and personality correlates of responses to the three scenarios. In general, people lied most in the academic scenario and least in the relationship scenario. Differences in lying efficacy, positive affect, and negative affect were modest. Cognitive load was somewhat lower in the relationship scenario. Correlations between the Dark Tetrad scenario responses were generally similar for professional and academic scenarios and somewhat weaker for the relationship scenario.

\subsection{Regression Models}

A set of regression models were estimated predicting propensity to lie (general, scenario average, professional scenario, academic scenario, and relationship scenario) from gender, age, and the Dark Tetrad. In general, demographics provided minimal prediction and the Dark Tetrad were strong predictors of propensity to lie. Prediction of 
propensity to lie was best in both the general tendency to lie and the scenario average. Machiavellianism was always the strongest predictor. Overall, whether it was predicting the stated general tendency or scenario average the coefficients were similar with narcissism and sadism both as significant predictors, although the general tendency also had a significant effect for psychopathy.

\section{Discussion}

In the current study, we used social exchange theory to understand the relationship between the Dark Tetrad traits and the propensity to lie across a number of important trust-dependent contexts. Several key findings emerged. First, the Dark Tetrad traits were positively associated with a greater propensity to lie, greater selfefficacy, and less cognitive load, more positive affect, and less negative affect while lying. Second, of the four traits, Machiavellianism was the strongest predictor of propensity to lie, and sadism provided incremental prediction. Third, when considering their propensity to lie, Dark Tetrad traits were more strongly related to the experience of elevated positive affect than they were with the experience of lower levels of negative affect. Finally, within the vignettes, it was lying efficacy followed by positive affect which best predicted a propensity to lie, as opposed to cognitive load or negative affect. The results have implications for understanding the effect of dark traits on deception and for the integration of sadism into dispositional models of lying.

\subsection{Dark Tetrad and Propensity to Lie}

Of the Dark Tetrad traits, Machiavellianism was the strongest predictor of willingness to deceive, although each trait was positively correlated with willingness to lie. This mirrors the findings of Baughman et al. (2014), who posited that trait Machiavellianism may be particularly important in deceptive contexts that can assist in outcomes such as social attainment and maneuvering. This seems highly apparent when contrasting the strength that trait Machiavellianism predicted a higher propensity to lie in both the professional and academic versus mating contexts. In contrast, we found narcissists had a more modest propensity to lie and were characterized more by a high, possibly inflated, sense of their own ability to lie. Interestingly, while psychopathy correlated with the propensity to lie in the vignettes, it did not provide incremental prediction in the regression models. Potentially, this lack of incremental prediction could be attributed to the shared variance between the traits, particularly the strong interrelationship between Machiavellianism and psychopathy $(r=.58)$. Machiavellianism and psychopathy are positively related and considered to characteristically overlap (McHoskey et al., 1998); however, Machiavellianism differs from psychopathy with its greater emphasis on more calculated, strategic antisocial tactics (Jones \& Paulhus, 2017).

\subsection{Emotions}

Interestingly, in addition to providing incremental prediction of propensity to lie, sadism exhibited a unique profile of vignette correlations. Sadism was particularly characterized by strong correlations with positive emotions when considering to lie consistent with many of the elements of schadenfreude and 'Dupers Delight' (Ekman, 2003). This is consistent with sadism being characterized by the self-satisfaction associated with the act of successfully lying and the pleasure of observing this act. Thus, while sadism and psychopathy were relatively highly correlated $(r=.58)$ and share 
some conceptual overlap, sadism was more aligned with the notion of pleasure when lying.

\subsection{Cognitive Load}

Cognitive load theory posits that lying requires more cognitive resources telling the truth (Vrij, Fisher, Mann, \& Leal, 2008). Results shows that not only were those high in Dark Tetrad traits more willing to lie, but they also reported less cognitive load when lying. When considered across each of the domain specific contexts, the lowest levels of cognitive load were observed in the relationship scenario compared to the academic and professional contexts. It may be that contextual factors including the risk level and the target of the deception explain this result (Jones \& Paulhus, 2017; Wissing $\&$ Renhard, 2019). For example, due to the familiarity of the target (i.e., partner), those high on the dark traits may have felt a heightened sense of confidence and lower perceived risk that their lie would not be believed. Alternatively, the consequences of being found to have lied may have been judged to be lower in comparison to the professional and academic contexts. This may therefore have translated to the reduced level of cognitive resources being perceived to be required to lie successfully in the relationship context utilized in the current study.

\subsection{Limitations}

Several limitations should be noted. First, responses to the vignettes used in the current study may not reflect actual behavior. Real life interactions introduce a range of contextual variables related to the risk level and target of the deceptive behavior required to be successful that may moderate the individual's motivation or propensity to lie. Second the sample had a greater representation of young adults, females, and students which may have implications for generalizing the current findings. Finally, to address the limitations associated with this cross-sectional design, future research could utilize a diary-based methodology to record daily deception and compare this in conjunction with dark trait personality scores.

\subsection{Conclusion}

This study found individuals high on the Dark Tetrad have a higher propensity to lie, feel more positive emotions as a result, and report that fewer cognitive resources are required to lie successfully across a number of important trust-based contexts. This highlights the dangers associated with human tendency to expect others to provide truthful statements in interpersonal interactions (Levine, 2020), especially in those intrinsically linked to processes where integrity and fairness are paramount. While the general need to assume others are being honest is a critical assumption that arguably underlies the functioning of society, this study's findings reinforce the need for thorough credibility assessments in trust-based contexts when dealing with individuals who exhibit the Dark Tetrad traits.

\section{References}

Abell, L., \& Brewer, G. (2014). Machiavellianism, self-monitoring, self-promotion and relational aggression on Facebook. Computers in Human Behavior, 36, 258-262.

Baughman, H. M., Jonason, P. K., Lyons, M., \& Vernon, P. A. (2014). Liar liar pants on fire: Cheater strategies linked to the dark triad. Personality and Individual Differences, 71, 35-38.

Bonaccio, S., Reeve, C. L., \& Lyerly, J. (2016). Academic entitlement: Its personality and general mental ability correlates, and academic consequences. Personality and Individual Differences, 102, 211-216.

Bond, C. F., \& DePaulo, B. M. (2006). Accuracy of deception judgments. Personality and Social Psychology Review, 10(3), 214234. 
Brewer, G., Hunt, D., James, G., \& Abell. L. (2015). Dark Triad traits, infidelity and romantic revenge. Personality and Individual Differences, 83, 122-127.

Brunell, A. B., Staats, S., Barden, J., \& Hupp, J. M. (2011). Narcissism and academic dishonesty: The exhibitionism dimension and the lack of guilt. Personality and Individual Differences, 50, 323-328.

Carpenter, C. J. (2012). Narcissism on Facebook: Self-promotional and anti-social behavior. Personality \& Individual Differences, $52,482-486$.

Chabrol, H., Bouvet, R., \& Goutaudier, N. (2017) The Dark Tetrad and antisocial behavior in a community sample of college students, Journal of Forensic Psychology Research and Practice, 17, 295-304.

Chabrol, H., Bronchain, J., Bamba, C. I. M., \& Raynal, P. (2020) The Dark Tetrad and radicalization: Personality profiles in young women, Behavioral Sciences of Terrorism and Political Aggression, 12, 157-168.

Chabrol, H., Leeuwen, N. V., Rodgers, R., \& Sejourne, N. (2009). Contributions of psychopathic, narcissistic, Machiavellian, and sadistic personality traits to juvenile delinquency. Personality and Individual Differences, 47. 734-739.

Chabrol, H., Melioli, T., Van Leeuwen, N., Rodgers, R., \& Goutaudier, N. (2015). The Dark Tetrad: Identifying personality profiles in high-school students. Personality and Individual Differences, 83, 97-101.

Cracker, N., \& March, E. (2016). The dark side of Facebook: The Dark Tetrad, negative social potency, and trolling behaviours. Personality and Individual Differences, 102, 79-84.

Cropanzano, R. \& Mitchell, M. S. (2005). Social Exchange Theory: An interdisciplinary review. Journal of Management, 31.

DePaulo, B., Kashy, D., Kirkendol, S., Wyer, M., \& Epstein, J. (1996). Lying in everyday life. Journal of Personality and Social Psychology. 70, 979-995.

DePaulo, B. M. (2019). Lying in social psychology. J. Meibauer (ed.), The oxford handbook of lying, (436-445), Oxford University Press

Ekman, P. (2001). Telling lies: Clues to deceit in the marketplace, marriage, and politics (3rd ed.). Norton.

George, J. F., \& Robb, A. (2008) Deception and computer-mediated communication in daily life. Communication Reports, 21, 92103.

Griffin, D. J., \& Bender, C. (2019). Culture and deception: The influence of language and societies on lying. T. Docan-Morgan (ed.), The palgrave handbook of deceptive communication, (67-89). Palgrave Macmillan.

Hardaker, C. (2010). Trolling in asynchronous computer-mediated communication: from user discussions to academic definitions. Journal of Politeness Research, 6, 215-242.

Jonason, P. K., \& Krause, L. (2013). The emotional deficits associated with the Dark Triad traits: Cognitive empathy, affective empathy, and alexithymia. Personality \& Individual Differences, 55, 532-537.

Jonason, P. K., Li, N. P., Webster, G. D., \& Schmitt, D. P. (2009). The Dark Triad: Facilitating a short-term mating strategy in men. European Journal of Personality, 23, 5-18

Jonason, P. K., Li, N. P., \& Teicher, E. A. (2010). Who is James Bond? The Dark Triad as an agentic social style. Individual Differences Research, 8, 111-120.

Jonason, P. K., Lyons, M., Baughman, H. M., \& Vernon, P. A. (2014). What a tangled web we weave: The Dark Triad and deception. Personality Individual. Differences. 70, 117-119.

Jonason, P. K., \& Webster, G. D. (2010). The Dirty Dozen: A concise measure of the Dark Triad. Psychological Assessment, 22, $420-432$.

Jonason, P. K., \& Webster, G. D. (2012). A protean approach to social influence: Dark Triad personalities and social influence tactics. Personality \& Individual Differences, 52, 521-526.

Jones, D. N., \& Paulhus, D. L. (2011). The role of impulsivity in the Dark Triad of personality. Personality and Individual Differences, 51, 679-682.

Jones, D. N., \& Paulhus, D. L. (2017). Duplicity among the Dark Triad: Three faces of deceit. Journal of Personality and Social Psychology, 113, 329-342.

Levine, T. (2020). Duped: Truth-Default Theory and the social science of lying and deception.University Alabama Press.

Marelich, W. D., Lundquist, J., Painter, K., \& Mechanic, M. B. (2008). Sexual deception as a social-exchange process: Development of a behavior-based sexual deception scale. Journal of Sex Research, 45, 27-35.

McHoskey, J., Worzel, W., \& Szyarto, C. (1998). Machiavellianism and psychopathy. Journal of Personality and Social Psychology, 74, 192-210.

Miller, J. D., \& Lynam, D. R. (2015). Understanding psychopathy using the basic elements of personality. Social and Personality Psychology Compass, 9, 223-237.

O'Boyle, E. H., Forsyth, D. R., Banks, G. C., \& McDaniel, M. A. (2012) A meta-analysis of the Dark Triad and work behavior: A social exchange perspective. Journal of Applied Psychology. 97, 557-79.

O'Meara, A., Davies, J., \& Hammond, S. (2011). The psychometric properties and utility of the Short Sadistic Impulse Scale (SSIS). Psychological assessment, 23, 523.

Paulhus, D. L., \& Williams, K. M. (2002). The Dark Triad of personality: Narcissism, Machiavellianism, and psychopathy. Journal of Research in Personality, 36, 556-563.

Porter, S., \& ten Brinke, L. (2010). The truth about lies: What works in detecting high-stakes deception? Legal and criminological Psychology, 15, 57-75. 
Raskin, R., Novacek, J., \& Hogan, R. (1991). Narcissistic self-esteem management. Journal of Personality and Social Psychology, 60, 911-918.

Rauthmann, J. F. (2012). The Dark Triad and interpersonal perception: Similarities and differences in the social consequences of narcissism, Machiavellianism, and psychopathy. Social Psychological and Personality Science, 3, 487-496.

Serota, K. B., \& Levine, T. R. (2015). A few prolific liars: Variation in the prevalence of lying. Journal of Language and Social Psychology. 34, 138-57.

Serota K. B., Levine T. R., \& Boster F. J. (2020) The prevalence of lying in America: Three studies of self-reported lies. Human Communication Research. 36, 2-25.

Venables, N. C., Hall, J. R., \& Patrick, C. J. (2014). Differentiating psychopathy from antisocial personality disorder: A triarchic model perspective. Psychological Medicine, 44, 1005-1013.

Vrij, A., Fisher, R., Mann, S., \& Leal, S. (2008). A cognitive load approach to lie detection. Journal of Investigative Psychology and Offender Profiling, 5, 39-43. Wissing, B. G., \& Reinhard, M-A. (2017). The Dark Triad and deception perceptions. Frontiers in Psychology, 10, 1-7.

Wright, G. R., Berry, C. J., Catmur, C., \& Bird, G. (2015). Good liars are neither 'dark' nor self-deceptive. PloS one, 10, 1-11.

\section{Table 1}

Correlation between Demographics, Dark Tetrad, General Lying Tendencies, and Average Responses to the Three Scenarios

\begin{tabular}{|c|c|c|c|c|c|c|c|c|c|c|c|c|c|c|}
\hline & $\mathrm{M}$ & SD & 1 & 2 & 3 & 4 & 5 & 6 & 7 & 8 & 9 & 10 & 11 & 12 \\
\hline \multicolumn{15}{|l|}{ Demographics } \\
\hline 1. Female & 0.60 & 0.49 & & & & & & & & & & & & \\
\hline 2. Age & 34.7 & 8.85 & .11 & & & & & & & & & & & \\
\hline \multicolumn{15}{|l|}{ Traits } \\
\hline 3. Machiavellianism & 3.34 & 0.74 & -.10 & -.12 & & & & & & & & & & \\
\hline 4. Narcissism & 2.56 & 0.70 & -.12 & .01 & .39 & & & & & & & & & \\
\hline 5. Psychopathy & 2.23 & 0.61 & -.13 & -.02 & .52 & .35 & & & & & & & & \\
\hline 6. Sadism & 1.87 & 0.83 & -.15 & -.02 & .51 & .26 & .58 & & & & & & & \\
\hline \multicolumn{15}{|l|}{ General Tendencies } \\
\hline 7. Propensity to Lie & 3.27 & 1.21 & -.03 & -.07 & .43 & .30 & .36 & .36 & & & & & & \\
\hline 8. Lying Efficacy & 5.47 & 1.05 & -.06 & .01 & .30 & .24 & .14 & .16 & .20 & & & & & \\
\hline \multicolumn{15}{|l|}{ Scenario Averages } \\
\hline 9. Propensity to Lie & 2.40 & 0.90 & -.04 & -.05 & .47 & .26 & .29 & .34 & .42 & .25 & & & & \\
\hline 10. Lying Efficacy & 2.69 & 0.94 & -.06 & .04 & .41 & .34 & .26 & .29 & .34 & .36 & .54 & & & \\
\hline 11. Cognitive Load & 3.46 & 0.56 & .08 & -.18 & -.20 & -.20 & -.29 & -.32 & -.27 & -.14 & -.11 & -.26 & & \\
\hline 12. Positive Affect & 1.33 & 0.55 & -.13 & -.03 & .42 & .39 & .32 & .42 & .35 & .17 & .36 & .34 & -.24 & \\
\hline 13. Negative Affect & 2.63 & 0.74 & .13 & .02 & -.16 & -.08 & -.16 & -.19 & -.13 & -.11 & -.14 & -.20 & .30 & -.11 \\
\hline
\end{tabular}

Note. $N=615$. Female is coded $0=$ Male, $1=$ Female. Absolute correlations larger than or equal to $.08, .11$, and .14 are significant at $.05, .01$, and .001 respectively. 
Table 2

Descriptive Statistics and Personality Correlates of Responses to Scenarios

\begin{tabular}{|c|c|c|c|c|c|c|c|c|}
\hline \multirow[b]{2}{*}{ Variable } & \multirow[b]{2}{*}{$M$} & \multirow[b]{2}{*}{$S D$} & \multicolumn{6}{|c|}{ Correlations } \\
\hline & & & Female & Age & Machiavellianism & Narcissism & $\begin{array}{l}\text { Psychopa } \\
\text { thy }\end{array}$ & Sadism \\
\hline \multicolumn{9}{|c|}{ Propensity to Lie } \\
\hline Professional & 2.74 & 1.37 & -.05 & -.06 & $.38 * * *$ & $.18 * * *$ & $.23 * * *$ & $.28 * * *$ \\
\hline Academic & 3.01 & 1.43 & -.05 & $-.08 *$ & $.39 * * *$ & $.19 * * *$ & $.23 * * *$ & $.28 * * *$ \\
\hline Relationship & 1.46 & 0.90 & .02 & .07 & $.24 * * *$ & $.21 * * *$ & $.15 * * *$ & $.15 * * *$ \\
\hline \multicolumn{9}{|l|}{ Lying Efficacy } \\
\hline Professional & 2.87 & 1.15 & $-.08 *$ & .05 & $.36 * * *$ & $.28 * * *$ & $.24 * * *$ & $.25 * * *$ \\
\hline Academic & 2.58 & 1.19 & -.07 & .00 & $.35 * * *$ & $.31 * * *$ & $.24 * * *$ & $.26 * * *$ \\
\hline Relationship & 2.62 & 1.42 & .01 & .04 & $.24 * * *$ & $.18 * * *$ & $.12 * * *$ & $.16 * * *$ \\
\hline \multicolumn{9}{|l|}{ Cognitive Load } \\
\hline Professional & 3.69 & 0.68 & $.09 *$ & $-.18 * * *$ & $-.17 * * *$ & $-.24 * * *$ & $-.25 * * *$ & $-.26 * * *$ \\
\hline Academic & 3.58 & 0.69 & $.10 * *$ & $-.12 * *$ & $-.19 * * *$ & $-.18 * * *$ & $-.27 * * *$ & $-.29 * * *$ \\
\hline Relationship & 3.13 & 0.73 & -.01 & $-.14 * * *$ & $-.11 * * *$ & $-.08 *$ & $-.19 * * *$ & $-.21 * * *$ \\
\hline \multicolumn{9}{|l|}{ Positive Affect } \\
\hline Professional & 1.41 & 0.73 & $-.16 * * *$ & -.03 & $.37 * * *$ & $.33 * * *$ & $.29 * * *$ & $.37 * * *$ \\
\hline Academic & 1.38 & 0.74 & $-.12 *$ & -.03 & $.39 * * *$ & $.34 * * *$ & $.29 * * *$ & $.39 * * *$ \\
\hline Relationship & 1.21 & 0.50 & -.02 & -.03 & $.24 * * *$ & $.29 * * *$ & $.19 * * *$ & $.26 * * *$ \\
\hline \multicolumn{9}{|l|}{ Negative Affect } \\
\hline Professional & 2.72 & 0.87 & $.17 * * *$ & -.01 & $-.14 * * *$ & $-.09 *$ & $-.14 * * *$ & $-.16 * * *$ \\
\hline Academic & 2.65 & 0.87 & $.13 *$ & .03 & $-.20 * * *$ & $-.09 *$ & $-.17 * * *$ & $-.19 * * *$ \\
\hline Relationship & 2.54 & 0.92 & .02 & .02 & $-.06 * * *$ & -.03 & $-.11 * *$ & $-.12 * *$ \\
\hline
\end{tabular}

Note. $N=615$. Female is coded $0=$ Male, $1=$ Female. Absolute correlations larger than or equal to $.08, .11$, and .14 are significant at $.05, .01$, and .001 respectively.

$* p<.05, * * p<.01, * * * p<.001$

Table 3

Regression Models Predicting Propensity to Lie in General and across Scenarios

\begin{tabular}{|c|c|c|c|c|c|}
\hline & General & Average & Professional & Academic & Relationship \\
\hline \multicolumn{6}{|l|}{ Standardized Beta } \\
\hline Female & 0.05 & 0.03 & 0.01 & 0.01 & 0.05 \\
\hline Age & -0.04 & -0.01 & -0.02 & -0.05 & $0.09 *$ \\
\hline Machiavellianism & $0.25 * * *$ & $0.38 * * *$ & $0.31 * * *$ & $0.31 * * *$ & $0.19 * * *$ \\
\hline Narcissism & $0.13 * * *$ & $0.08 *$ & 0.03 & 0.05 & $0.13 * *$ \\
\hline Psychopathy & $0.10^{*}$ & -0.02 & -0.02 & -0.01 & 0.00 \\
\hline Sadism & $0.15 * * *$ & $0.14 * *$ & $0.13 * *$ & $0.12 *$ & 0.03 \\
\hline Adjusted $\mathrm{R}^{2}$ & .23 & .24 & .15 & .15 & .07 \\
\hline
\end{tabular}




\section{Online Supplement}

\subsection{Scenario 1 Mating Context}

You and your partner have been in a healthy relationship for over two years. One day, you receive an email from your ex-partner asking if you want to meet up for coffee. Although hesitant at first, you agree to meet with them. You decide not to tell your current partner about this meeting. While you are having coffee at the cafe with your ex-partner, you notice that a friend of your current partner is looking at you from across the room. Instead of approaching you to say hello, this friend walks out of the café. When you return home, your partner says that they heard from a friend about your meeting with your ex and demands to know your side of the story.

\subsection{Scenario 2 Academic Context}

It is near to the end of the semester and you have assignments building up and exams to study for. One important assignment is due in and you have yet to write it. The marks for the assignment count for $40 \%$ of your overall grade for this course and it is not your best subject. You are friends with a student who took the same course the year before you and they offer to give you their assignment to help you out. They got $82 \%$ for their essay. You finish the assignment and hand it in on time, although you have plagiarized a substantial amount of your friend's work. You then think nothing more about it. When the assignments have been marked, your lecturer asks to have a word with you about some of the assignment he recognizes. He suspects you have plagiarized but you tell him that the essay was all your own work.

\subsection{Scenario 3 Professional Context}

You were eager to acquire a job at a large well-known organization. You applied for the job previously but didn't receive an interview based upon your inadequate experience. Some time goes by and another job advertisement comes up at the same organization for a similar position. You reapply after adding some more fictional qualifications and experiences to your resume. Based upon your resume you are called in for an interview, in which you do very well and are offered a position - that you immediately accept. You think nothing of this and settle into your new job. Not long after, another staff member who you previously worked with identified that you lied on your resume. Your boss demands that you assure him that you are qualified.

\section{Demographic details}

Participants resided in the United States (47\%), United Kingdom (11\%), Australia (11\%), Canada (7\%), Germany (3.4\%), Netherlands (1.8\%), France (1.4\%), New Zealand (1.3\%), Finland (1.1\%), Sweden (1.0\%) and other countries (14\%). Of the sample, 39.\% were students, 47\% were employed but not students, $9 \%$ were unemployed, $1 \%$ were retired, and $4 \%$ were other. Education level was as follows: less than high school completion (3\%), completed high school (31\%), certificate or diploma $(15 \%)$, undergraduate degree $(36 \%)$, masters or $\mathrm{PhD}(15 \%)$. Relationship status was as follows: Married (18.7\%), Widowed (0.3\%), Single (55.8\%), Separated (1.3\%), Defacto $(8.3 \%)$, Other $(15.6 \%)$. 